\title{
T cell receptor signaling pathway and cytokine-cytokine receptor interaction affect the rehabilitation process after respiratory syncytial virus infection
}

\author{
Zuanhao Qian ${ }^{\text {Corresp., Equal first author, 1 }}$, Zhenglei Zhang ${ }^{\text {Equal first author, 1 }}$, Yingying Wang ${ }^{1}$ \\ ${ }^{1}$ Department of Pediatrics, Taikang Xianlin Drum Tower Hospital, Nanjing, China \\ Corresponding Author: Zuanhao Qian \\ Email address: qianzuanhao@163.com
}

Background: Respiratory syncytial virus (RSV) is the main cause of respiratory tract infection, which seriously threatens the health and life of children. This study is conducted to reveal the rehabilitation mechanisms of RSV infection. Methods: E-MTAB-5195 dataset was downloaded from EBI ArrayExpress database, including 39 acute phase samples in the acute phase of infection and 21 samples in the recovery period. Using limma package, differentially expressed RNAs (DE-RNAs) were analyzed. The significant modules were identified using WGCNA package, and the mRNAs in them were conducted with enrichment analysis using DAVID tool. Afterwards, co-expression network for the RNAs involved in the significant modules was built by Cytoscape software. Additionally, RSV-correlated pathways were searched from Comparative Toxicogenomics Database, and then pathway network was constructed. Results: There were 2489 DE-RNAs between the two groups, including 2386 DE-mRNAs and 103 DE-IncRNAs. The RNAs in the black, salmon, blue, tan and turquoise modules correlated with time point were taken as RNA set1. Meanwhile, the RNAs in brown, blue, magenta and pink modules related to staging were defined as RNA set2. In the pathway networks, CD4OLG and RASGRP1 co-expressed with LINC00891/LINC00526/LINC01215 were involved in T cell receptor signaling pathway, and ILIB, ILIR2, IL18, and IL18R1 co-expressed with BAIAP2-AS1/CRNDE/LINC01503/SMIM25 were implicated in cytokine-cytokine receptor interaction. Conclusion: LINC00891/LINC00526/LINC01215 co-expressed with CD40LG and RASGRP1 might affect the rehabilitation process of RSV infection through $T$ cell receptor signaling pathway. Besides, BAIAP2-AS1/CRNDE/LINC01503/SMIM25 co-expressed with IL1 and IL18 families might function in the clearance process after RSV infection via cytokine-cytokine receptor interaction. 
1 T cell receptor signaling pathway and cytokine-cytokine receptor interaction affect the rehabilitation

2 process after respiratory syncytial virus infection

3 Running title: Rehabilitation by RSV infection

4

5 Zuanhao Qian ${ }^{\ddagger}$, , Zhenglei Zhang', Yingying Wang

6 Department of Pediatrics, Taikang Xianlin Drum Tower Hospital, No.188, Lingshan North Road, Qixia

7 District, Nanjing, China

8

9 ‡ equal contribution

11 Corresponding author:

Zuanhao Qian, E-mail: qianzuanhao@163.com; Address: Taikang Xianlin Drum Tower Hospital, No.188,

Lingshan North Road, Qixia District, Nanjing, 210046, China.

Abstract

Background: Respiratory syncytial virus (RSV) is the main cause of respiratory tract infection, which seriously

threatens the health and life of children. This study was conducted to reveal the rehabilitation mechanisms of

RSV infection.

Methods: E-MTAB-5195 dataset, including 39 acute phase samples in the acute phase of infection and 21 
differentially expressed RNAs (DE-RNAs) were analyzed. The modules were identified using WGCNA

package, and the mRNAs in modules were conducted with enrichment analysis using DAVID tool. Afterwards,

co-expression network for the RNAs involved in the significant modules was built by Cytoscape software.

Additionally, RSV-correlated pathways were searched from Comparative Toxicogenomics Database, and then

pathway network was constructed.

Results: There were 2489 DE-RNAs between the two groups, including 2386 DE-mRNAs and 103 DE-

IncRNAs. The RNAs in the black, salmon, blue, tan and turquoise modules correlated with time point were

taken as RNA set1. The RNAs in brown, blue, magenta and pink modules related to staging were defined as

RNA set2. In the pathway networks, CD40LG and RASGRP1 co-expressed with

LINC00891/LINC00526/LINC01215 were involved in T cell receptor signaling pathway, and IL1B, IL1R2,

IL18, and IL18R1 co-expressed with BAIAP2-AS1/CRNDE/LINC01503/SMIM25 were implicated in cytokine-

cytokine receptor interaction.

Conclusion: LINC00891/LINC00526/LINC01215 co-expressed with CD40LG and RASGRP1 might affect the

rehabilitation process of RSV infection through $\mathrm{T}$ cell receptor signaling pathway. Besides, BAIAP2-

AS1/CRNDE/LINC01503/SMIM25 co-expressed with IL1 and IL18 families might function in the clearance

process after RSV infection via cytokine-cytokine receptor interaction.

\section{Introduction}

Respiratory syncytial virus (RSV) belongs to the paramyxovirus family of pneumonia genus, which is the 
therapy, fogging machine antivirus therapy, and antibacterial drug treatment (Handforth et al. 2004).

Approximately $60 \%$ of infants will be infected with RSV during their first season of the virus, and almost all children experience RSV infection at the age of 2-3 years old in the United States (Hama et al. 2015; Louis et al. 2016). Therefore, exploring the possible mechanisms of RSV infection is of great importance.

Through specific antagonistic effects on host protein functions, induction of RNA stress particles and induction of changes in host gene expression patterns, RSV can change the transcription of host genes and the translation of host transcripts (Tripp et al. 2013). Long noncoding RNAs (lncRNAs) are a class of RNAs with a length greater than $200 \mathrm{nt}$ and lacking the ability to encode proteins (Andreia et al. 2015). At present, lncRNAs are found to have complex functions and can be involved in all stages of gene expression regulation (Wahlestedt 2013; Wang et al. 2011). LncRNA maternally expressed 3 (MEG3) expression is decreased in RSV-infected nasopharyngeal samples, and $M E G 3$ can suppress RSV infection via inhibiting toll-like receptor 4 (TLR4) signaling (Tao et al. 2018). Through mediating E2F transcription factor 3 (E2F3) expression, lncRNA plasmacytoma variant translocation $1(P V T 1)$ is deemed to be correlated with the effects of $\alpha$-asarone in the treatment of RSV-induced asthma (Yu et al. 2017). Additionally, transcription elongation is involved in IFN-stimulated gene (ISG) expression induced by RSV, and cyclin-dependent kinase $9(C D K 9)$ activity may serve as a potential target for immunomodulation in RSV-associated lung disease (Tian et al. 2013). Nevertheless, the pathogenesis of RSV infection has not been fully understood.

In 2016, Jong et al performed a blood transcriptome profiling analysis and identified an 84 gene signature that could predict the course of RSV disease (Jong et al. 2016). However, they had not performed indepth bioinformatics analyses to explore the rehabilitation mechanisms of RSV infection. Using the dataset deposited by Jong et al (Jong et al. 2016), the lncRNA and mRNA expression profiles of RSV-infected blood 
samples from infants were compared to screen the molecules markers between acute phase and recovery period of RSV infection. This study might contribute to investigating the gene expression changes in blood from acute phase to recovery period of RSV infection and revealing the underlying mechanisms of disease rehabilitation.

\section{Materials and Methods}

\section{Data source and data preprocessing}

The E-MTAB-5195 dataset was acquired from EBI ArrayExpress database
(https://www.ebi.ac.uk/arrayexpress/) (Brazma et al. 2003), which was detected on the platform of Affymetrix GeneChip Human Genome U133 Plus 2.0. E-MTAB-5195 contained 60 blood samples from infants, including 39 samples in the acute phase of infection (14 females and 25 males; mean age $=146.49$ days $)$ and 21 samples in the recovery period ( 11 females and 10 males; mean age $=137.95$ days). This study analyzed the expression profile dataset obtained from public database, and no animal or human experiments were involved. Therefore, no ethical review or informed consents were needed.

The raw expression profile data in .CEL format were conducted with format transformation, filling of missing data (median method), background correction (MicroArray Suite method), and data standardization (quantile method) using the $\mathrm{R}$ package oligo (version 1.41, http://www.bioconductor.org/packages/release/bioc/html/oligo.html) (Parrish \& Spenceriii 2004).

\section{Differential expression analysis}

The platform information of E-MTAB-5195 was downloaded. Combined with the Transcript ID, RefSeq ID, and location information provided in platform information, the mRNAs and lncRNAs in the expression profile E-MTAB-5195 were annotated based on the 19198 protein coding genes and 3909 lncRNAs included 
in HUGO Gene Nomenclature Committee (HGNC, http://www.genenames.org/) database (Bruford et al. 2008).

infection and samples in the recovery period. Using the $\mathrm{R}$ package limma (version 3.34.0, https://bioconductor.org/packages/release/bioc/html/limma.html) (Ritchie et al. 2015), differential expression analysis for the two groups were carried out. The false discovery rate (FDR) $<0.05$ and $\mid \log$ fold change (FC) $\mid>$ 0.263 were defined as the criteria for screening differentially expressed RNAs (DE-RNAs). Based on the expression levels of the screened RNAs, the $\mathrm{R}$ package pheatmap (version 1.0.8, https://cran.rproject.org/package=pheatmap) (Wang et al. 2014) was used to perform hierarchical clustering analysis (Szekely \& Rizzo 2005) and present the result in clustering heatmap.

\section{Weighed gene co-expression network analysis (WGCNA) and enrichment analysis}

The systems biology method WGCNA can be applied for integrating gene expression and identify disease-associated modules ( $\mathrm{Li}$ et al. 2018). Using the $\mathrm{R}$ package WGCNA (version 1.61, https://cran.rproject.org/web/packages/WGCNA/) (Langfelder \& Horvath 2008), the DE-RNAs were analyzed to identify the modules significantly related to disease states and clinical factors. The analysis procedures included assumption of the scale-free network, definition of co-expression correlation matrix, definition of adjacency function, calculation of dissimilarity coefficients, and identification of disease-associated modules. The modules having significant correlation with disease symptoms $(\mathrm{p}$-value $<0.05)$ and higher correlation coefficients compared with the control (grey module) were taken as the significant modules.

Then, DAVID online tool (version 6.8, https://david.ncifcrf.gov/) (Lempicki 2008) was utilized to perform Gene Ontology (GO) (Consortium 2015) and Kyoto Encyclopedia of Genes and Genomes (KEGG) (Kanehisa et al. 2016) enrichment analyses for the mRNAs in the significant modules. The p-value $<0.05$ was 
selected as the threshold of enrichment significance.

\section{Co-expression network analysis}

For the lncRNAs and mRNAs in the significant modules, pearson correlation coefficients (PCCs) (Hauke \& Kossowski 2011) were calculated using the cor function in $\mathrm{R}$ (http://77.66.12.57/R-help/cor.test.html). Afterwards, visualization of co-expression network was conducted using Cytoscape software (http://www.cytoscape.org/) (Kohl et al. 2011). Moreover, KEGG pathways were enriched for the mRNAs in the co-expression network using DAVID tool (Lempicki 2008).

\section{Network analysis for the RSV-correlated pathways}

Using "Respiratory Syncytial Virus" as keyword, the KEGG pathways directly related to RSV were searched form Comparative Toxicogenomics Database (2017 update, http://ctd.mdibl.org/) (Davis et al. 2013).

By comparing the searched pathways and the pathways enriched for the mRNAs in the co-expression network, the overlapped pathways and the mRNAs involved in them were obtained. Furthermore, the clusters (including both IncRNAs and mRNAs) of the RNAs involved in the RSV-correlated pathways were extracted from the co-expression network to construct the network of RSV-correlated pathways.

\section{Results}

\section{Differential expression analysis}

The raw data of expression profile dataset E-MTAB-5195 were preprocessed (Figure 1). After annotation was performed based on platform information, a total of 16984 protein coding RNAs and 939 lncRNAs were obtained. Then, the samples were grouped according to disease stage information. There were a total of 2489 DE-RNAs between the two groups, including 2386 DE-mRNAs (1393 up-regulated and 993 down-regulated) and 103 DE-lncRNAs (62 up-regulated and 41 down-regulated) (Figure 2). The clustering heatmap of the DE- 
RNAs indicated that the DE-RNAs could separate the two groups of samples and thus had sample characteristics (Figure 3).

\section{WGCNA and enrichment analysis}

The 2489 DE-RNAs were further analyzed and screened using WGCNA. In order to satisfy the premise of scale-free network distribution as far as possible, the value of the weight parameter "power" of adjacency matrix needed to be explored. The selection range of network construction parameters was set, and the scalefree topological matrix was calculated. The value of "power" when the square value of the correlation coefficient reached 0.9 for the first time was selected, namely, power $=18$. At this time, the average node degree of the constructed co-expression network was 1 , which fully conformed to the properties of small world network (Figure 4A). Then, the dissimilarity coefficients between the gene points were calculated, and the system cluster tree was obtained. The minimum number of genes was set as 70 for each module, and the pruning height was set as cutHeight $=0.99$. A total of 13 modules (except of grey module) (Figure 4B), and the number and type of RNAs involved in each module were listed in Table 1. Finally, the correlation between each module and different disease symptoms were calculated. The black, salmon, blue, tan and turquoise modules were significantly correlated with timepoint (acute/recovery), meanwhile, brown, blue, magenta and pink modules were significantly related to staging (Mild/Moderate/Severe) (Figure 4C). The RNAs in black, salmon, blue, tan and turquoise modules were taken as RNA set1 correlated with timepoint. Similarly, the RNAs in brown, blue, magenta and pink modules were considered as RNA set2 associated with staging. The mRNAs in RNA set1 and RNA set2 separately were conducted with enrichment analysis. For the mRNAs in RNA set1, 17 GO terms (such as regulation of transcription and lymphocyte activation) and 7 KEGG pathways (such as cell adhesion molecules (CAMs) and primary immunodeficiency) were enriched. 
148 For the mRNAs in RNA set2, 20 GO terms (including defense response and response to wounding) and 8

149 KEGG pathways (including cytokine-cytokine receptor interaction and lysosome) were predicted (Table 2).

150

151

152

153

\section{Co-expression network analysis}

For the RNA set1 and RNA set2, the PCCs between the lncRNAs and mRNAs separately were calculated and the lncRNA-mRNA pairs with PCC $>0.6$ were remained. Subsequently, the co-expression networks for RNA set1 (Figure 5A) and RNA set2 (Figure 5B) separately were constructed. In the co-expression network for RNA set1, there were 813 nodes (including 777 mRNAs and 36 lncRNAs) and 3773 edges. In the coexpression network for RNA set2, there were 529 nodes (including 521 mRNAs and 8 lncRNAs) and 967 edges. Moreover, 10 (such as hematopoietic cell lineage and primary immunodeficiency) and 11 (such as glutathione metabolism and cytokine-cytokine receptor interaction) KEGG pathways separately were enriched for the mRNAs in the co-expression networks for RNA set1 and RNA set2 (Table 3).

\section{Network analysis for the RSV-correlated pathways}

Based on Comparative Toxicogenomics Database, 44 KEGG pathways directly related to RSV were obtained. After comparing the searched pathways and the pathways enriched for the mRNAs in the coexpression networks, four (including hsa04660:T cell receptor signaling pathway, involving CD40 ligand (CD40LG) and RAS guanyl releasing protein 1 (RASGRP1); hsa04514:Cell adhesion molecules (CAMs); hsa04650:Natural killer cell mediated cytotoxicity; and hsa04350:TGF-beta signaling pathway) and two (including hsa04060:Cytokine-cytokine receptor interaction, involving interleukin 1 beta (IL1B), interleukin 1 receptor type II (IL1R2), interleukin 18 (IL18) and interleukin 18 receptor 1 (IL18R1); and hsa04621:NOD-like receptor signaling pathway) overlapped pathways separately were obtained for the mRNAs in the coexpression networks for RNA set1 and RNA set2. 
RNA set1, long intergenic non-protein coding RNA 891 (LINC00891), long intergenic non-protein coding

RNA 526 (LINC00526) and long intergenic non-protein coding RNA 1215 (LINC01215) were co-expressed

with CD4OLG, and LINC01215 was co-expressed with RASGRP1. Moreover, long intergenic non-protein

coding RNA 1503 (LINC01503) and SMIM25 co-expressed with IL1B, colorectal neoplasia differentially

expressed (CRNDE) co-expressed with IL1R2, BAIAP2 antisense RNA 1 (BAIAP2-AS1) and CRNDE co-

expressed with $I L 18$, and SMIM25 co-expressed with $I L 18 R I$ were involved in the pathway network for RNA

set2.

177

178

\section{Discussion}

In this study, 2489 DE-RNAs between the two groups of samples were obtained, including 2386 DEmRNAs (1393 up-regulated and 993 down-regulated) and 103 DE-lncRNAs (62 up-regulated and 41 downregulated). The black, salmon, blue, tan and turquoise modules were significantly correlated with timepoint, and the RNAs in them were taken as RNA set1. Meanwhile, brown, blue, magenta and pink modules were significantly related to staging, and the RNAs in these modules were considered as RNA set2. In the pathway network for RNA set1, LINC00891, LINC00526 and LINC01215 were co-expressed with CD40LG, and LINC01215 was co-expressed with RASGRP1. In the pathway network for RNA set2, LINC01503 and SMIM25 co-expressed with $I L 1 B, C R N D E$ co-expressed with IL1R2, BAIAP2-AS1 and CRNDE co-expressed with $I L 18$, and SMIM25 co-expressed with IL18RI were found.

After RSV infection, a large number of cytokines, chemokines, reactive oxygen species and other active mediators are induced in cells (Hansdottir et al. 2010). Natural immunity and regulatory immune responses are activated by identifying the conserved structures of corresponding antigens in bacteria, viruses and the 
190

191

192

193

194

195

196

197

198

199

200

environment (Shayakhmetov 2010; Sigurs et al. 2000). Immune response in the body changes dynamically all the time during viral infection (Thompson et al. 2011). This study analyzed the blood samples from infants in acute phase or recovery period of RSV infection, which was the clearance process after virus infection. After removing the virus in vivo, apoptosis of the effector $\mathrm{T}$ cells occurred and immune response of the $\mathrm{T}$ cells decreased (Jie et al. 2011).

In the pathway network for RNA set1, CD40LG and RASGRP1 were involved in T cell receptor signaling pathway in our present study. CD $40 L G$ promotes virus clearance ability of DNA vaccines encoding the $\mathrm{F}$ glycoprotein and enhances the immune response to RSV infection, therefore, CD4OLG can strengthen the durability of DNA vaccines against RSV infection (Harcourt et al. 2003). RASGRPI deficiency is related to reduced extracellular signal-regulated kinase $(E R K)$ phosphorylation in $\mathrm{B}$ cells and $\mathrm{T}$ cells, and can lead to defective proliferation, motility, and activation of the cells (Salzer et al. 2016). Therefore, the fact that LINC00891, LINC00526 and LINC01215 co-expressed with CD40LG, as well as LINC01215 co-expressed with RASGRP1 in our study might suggest the potential function of them in the rehabilitation mechanisms of RSV infection via $\mathrm{T}$ cell receptor signaling pathway.

In the pathway network for RNA set2, IL1B, IL1R2, IL18, and IL18R1 were implicated in cytokinecytokine receptor interaction in our study. Proinflammatory cytokines are found to be associated with the progression of cerebral white matter injury (WMI) in preterm infants, and cytokine-receptor interaction may be critical in determining the effects of inflammation in the development of the disease (Bass et al. 2008). IL18 is a cytokine that can enhance antiviral immunity and decrease viral load, while the RSV/IL18 recombinant deteriorates pulmonary viral infection (Harker et al. 2010). RSV-induced bronchiolitis is correlated with the occurrence of atopic and allergy asthma, and IL12 and IL18 play critical roles in Th1 and/or Th2 immune 

of RSV infection.

\section{Conclusions}

In conclusion, a total of 2489 DE-RNAs between the acute and recovery phase of RSV infection were screened. We identified that LINC00891, LINC00526 and LINC01215 co-expressed with CD40LG, as well as LINC01215 co-expressed with RASGRP1 might affect the rehabilitation process of RSV infection through $\mathrm{T}$ cell receptor signaling pathway. Furthermore, BAIAP2-AS1, CRNDE, LINC01503 and SMIM25 co-expressed with the cytokines in $I L 1$ and $I L 18$ families might act in the rehabilitation mechanisms of RSV infection via be further validated by experimental researches.

cytokine-cytokine receptor interaction. However, these findings obtained from bioinformatics analyses should

\section{References}

Andreia S, Marc B, and George C. 2015. The Clinical Relevance of Long Non-Coding RNAs in Cancer. Cancers 7:2169-2182.

Bass WT, Buescher ES, Hair PS, White LE, Welch JC, and Burke BL. 2008. Proinflammatory cytokinereceptor interaction model improves the predictability of cerebral white matter injury in preterm infants. Amer J Perinatol 25:211-218. 
Brazma A, Parkinson H, Sarkans U, Shojatalab M, Vilo J, Abeygunawardena N, Holloway E, Kapushesky M, Kemmeren P, and Lara GG. 2003. ArrayExpress - a public repository for microarray gene expression data at the EBI. Nucleic Acids Research 31:68-71.

Bruford EA, Lush MJ, Wright MW, Sneddon TP, Povey S, and Birney E. 2008. The HGNC Database in 2008: a resource for the human genome. Nucleic Acids Research 36:D445.

Chang CH, Huang Y, and Anderson R. 2003. Activation of vascular endothelial cells by IL-1alpha released by epithelial cells infected with respiratory syncytial virus. Cellular Immunology 221:37-41.

Consortium TGO. 2015. Gene Ontology Consortium: going forward. Nucleic Acids Research 43:1049-1056.

Davis AP, Murphy CG, Johnson R, Lay JM, Lennonhopkins K, Saracenirichards C, Sciaky D, King BL, Rosenstein MC, and Wiegers TC. 2013. The Comparative Toxicogenomics Database: update 2013. Nucleic Acids Research 41:D1104-D1114.

Fauroux B, Simões EAF, Checchia PA, Paes B, Figuerasaloy J, Manzoni P, Bont L, and Carbonellestrany X. 2017. The Burden and Long-term Respiratory Morbidity Associated with Respiratory Syncytial Virus Infection in Early Childhood. Infectious Diseases \& Therapy 6:173-197.

Hall CB, Weinberg GA, Iwane MK, Blumkin AK, Edwards KM, Staat MA, Auinger P, Griffin MR, Poehling KA, and Erdman D. 2009. The burden of respiratory syncytial virus infection in young children. New England Journal of Medicine 360:588.

Hama I, Takahashi S, Nakamura T, Ito Y, Kawasaki K, and Sago H. 2015. Risk of respiratory syncytial virus infection in infants with congenital cystic lung disease. Pediatrics International 57:253-257.

Handforth J, Sharland M, and Friedland JS. 2004. Prevention of respiratory syncytial virus infection in infants: Palivizumab is effective but too expensive, and vaccines are unavailable as yet. Bmj British Medical Journal 328:1026-1027.

Hansdottir S, Monick MM, Lovan N, Powers L, Gerke A, and Hunninghake GW. 2010. Vitamin D decreases respiratory syncytial virus induction of NF-kB-linked chemokines and cytokines in airway epithelium while maintaining the antiviral state. Journal of Immunology 184:965-974.

Harcourt JL, Brown MP, Anderson LJ, and Tripp RA. 2003. CD40 ligand (CD154) improves the durability of respiratory syncytial virus DNA vaccination in BALB/c mice. Vaccine 21:2964-2979.

Harker JA, Godlee A, Wahlsten JL, Lee DCP, Thorne LG, Sawant D, Tregoning JS, Caspi RR, Bukreyev A, and Collins PL. 2010. Interleukin 18 Coexpression during Respiratory Syncytial Virus Infection Results in Enhanced Disease Mediated by Natural Killer Cells. Journal of Virology 84:4073.

Hauke J, and Kossowski T. 2011. Comparison of Values of Pearson's and Spearman's Correlation Coefficients on the Same Sets of Data. Quaestiones Geographicae 30:87-93.

Jie S, Amber C, Sharma AK, Laubach VE, Jack RS, Werner M, and Braciale TJ. 2011. Autocrine Regulation of Pulmonary Inflammation by Effector T-Cell Derived IL-10 during Infection with Respiratory Syncytial Virus. Plos Pathogens 7:e1002173.

Jong VL, Ahout IML, Ham HJVD, Jans J, Zaaraouiboutahar F, Zomer A, Simonetti E, Bijl MA, Brand HK, and Ijcken WFJV. 2016. Transcriptome assists prognosis of disease severity in respiratory syncytial virus infected infants. Sci Rep 6:36603.

Kanehisa M, Sato Y, Kawashima M, Furumichi M, and Tanabe M. 2016. KEGG as a reference resource for gene and protein annotation. Nucleic Acids Research 44:D457-D462.

Kohl M, Wiese S, and Warscheid B. 2011. Cytoscape: software for visualization and analysis of biological 
networks. Methods Mol Biol 696:291-303.

Langfelder P, and Horvath S. 2008. WGCNA: an R package for weighted gene co-expression network analysis. BMC Bioinformatics 9:559.

Lempicki RA. 2008. Systematic and integrative analysis of large gene lists using DAVID bioinformatics resources : Abstract : Nature Protocols. Nature Protocols 4:44-57.

Li J, Zhou D, Qiu W, Shi Y, Yang JJ, Shi C, Wang Q, and Hui P. 2018. Application of Weighted Gene Coexpression Network Analysis for Data from Paired Design. Scientific Reports 8:622.

Louis B, Checchia PA, Brigitte F, Josep FA, Paolo M, Bosco P, Simões EAF, and Xavier CE. 2016. Defining the Epidemiology and Burden of Severe Respiratory Syncytial Virus Infection Among Infants and Children in Western Countries. Infectious Diseases \& Therapy 5:271-298.

Parrish RS, and Spenceriii HJ. 2004. Effect of Normalization on Significance Testing for Oligonucleotide Microarrays. Journal of Biopharmaceutical Statistics 14:575-589.

Patel JA, Jiang Z, Nakajima N, and Kunimoto M. 2010. Autocrine regulation of interleukin-8 by interleukin1 alpha in respiratory syncytial virus-infected pulmonary epithelial cells in vitro. Immunology 95:501506.

Ritchie ME, Phipson B, Wu D, Hu Y, Law CW, Shi W, and Smyth GK. 2015. limma powers differential expression analyses for RNA-sequencing and microarray studies. Nucleic Acids Research 43:e47.

Salzer E, Cagdas D, Hons M, Mace EM, Garncarz W, Â-Y P, Platzer R, Pfajfer L, Bilic I, and Ban SA. 2016. RASGRP1 deficiency causes immunodeficiency with impaired cytoskeletal dynamics. Nature Immunology 17:1352-1360.

Shayakhmetov DM. 2010. Virus Infection Recognition and Early Innate Responses to Non-Enveloped Viral Vectors. Viruses 2:244-261.

Sigurs N, Bjarnason R, Sigurbergsson F, and Kjellman B. 2000. Respiratory Syncytial Virus Bronchiolitis in Infancy Is an Important Risk Factor for Asthma and Allergy at Age 7. American Journal of Respiratory \& Critical Care Medicine 161:1501.

Szekely GJ, and Rizzo ML. 2005. Hierarchical Clustering via Joint Between-Within Distances: Extending Ward's Minimum Variance Method. Journal of Classification 22:151-183.

Tao XW, Zeng LK, Wang HZ, and Liu HC. 2018. LncRNA MEG3 ameliorates respiratory syncytial virus infection by suppressing TLR4 signaling. Molecular Medicine Reports:4138-4144.

Thompson MR, Kaminski JJ, Kurt-Jones EA, and Fitzgerald KA. 2011. Pattern Recognition Receptors and the Innate Immune Response to Viral Infection. Viruses 3:920-940.

Tian B, Zhao Y, Kalita M, Edeh CB, Paessler S, Casola A, Teng MN, Garofalo RP, and Brasier AR. 2013. CDK9-Dependent Transcriptional Elongation in the Innate Interferon-Stimulated Gene Response to Respiratory Syncytial Virus Infection in Airway Epithelial Cells. Journal of Virology 87:7075-7092.

Tripp RA, Mejias A, and Ramilo O. 2013. Host gene expression and respiratory syncytial virus infection. Current Topics in Microbiology \& Immunology 372:193-209.

Wahlestedt C. 2013. Targeting long non-coding RNA to therapeutically upregulate gene expression. Nature Reviews Drug Discovery 12:433.

Wang KC, Yang YW, Liu B, Sanyal A, Corceszimmerman R, Chen Y, Lajoie BR, Protacio A, Flynn RA, and Gupta RA. 2011. A long noncoding RNA maintains active chromatin to coordinate homeotic gene expression. Nature 472:120-124. 
316

317

318

319

320

321

322

323

324

325
Wang L, Cao C, Ma Q, Zeng Q, Wang H, Cheng Z, Zhu G, Qi J, Ma H, and Nian H. 2014. RNA-seq analyses of multiple meristems of soybean: novel and alternative transcripts, evolutionary and functional implications. BMC Plant Biology, 14,1(2014-06-17) 14:169.

Wang SZ, Bao YX, Rosenberger CL, Tesfaigzi Y, Stark JM, and Harrod KS. 2004. IL-12p40 and IL-18 modulate inflammatory and immune responses to respiratory syncytial virus infection. Journal of Immunology 173:4040-4049.

Yu X, Zhe Z, Tang B, Li S, Tang L, Wu Y, Chen X, and Fang H. 2017. $\alpha$-Asarone suppresses the proliferation and migration of ASMCs through targeting the lncRNA-PVT1/miR-203a/E2F3 signal pathway in RSV-infected rats. Acta Biochimica et Biophysica Sinica 49:598-608. 
326

327

328

329

330

331

332

\section{Figure legends:}

Figure 1. The box plots before and after data normalization.

Figure 2. The volcano plot of the differentially expressed RNAs (DE-RNAs). The green horizontal dashed line represents false discovery rate $(\mathrm{FDR})<0.05$, the two green vertical dashed lines represent $\mid \log$ fold change (FC) $\mid>0.263$. The blue and red dots separately represent the significantly down-regulated and up-regulated RNAs in the samples in the acute phase of infection.

Figure 3. The lustering heatmap based on the expression levels of the differentially expressed RNAs (DERNAs). Brown and blue in the sample bar represent the samples in the acute phase of infection and the samples in the recovery period, respectively. Yellow and blue in the heatmap notes up-regulation and downregulation, respectively.

Figure 4. The results of weighed gene co-expression network analysis (WGCNA). (A) The selection of the weight parameter "power" of adjacency matrix (left diagram; red line is the standard line when the square value of the correlation coefficient achieved 0.9 ) and the mean connectivity of RNAs (right diagram; red line indicates that the average node degree of the constructed co-expression network is 1 when power $=18$ ); (B) The tree diagram for module division (different color represent different modules); (C) The correlation heatmap for each module and different disease symptoms (the numbers in the grids represent correlation coefficients, and the numbers in parentheses represent the significance p-values). Yellow and green notes positive and negative correlation, respectively.

Figure 5. Co-expression networks. (A) The co-expression network for RNA set1. (B) The co-expression network for RNA set2. Blue and green represent RNAs that are significantly down-regulated in the samples in the acute phase of infection. Red and orange represent RNAs that are significantly up-regulated in the samples 
347 in the acute phase of infection. Squares and circles separately represent long non-coding RNAs (lncRNAs) and 348 mRNAs.

349 Figure 6. Networks of respiratory syncytial virus (RSV)-correlated pathways. (A) The network of RSVcorrelated pathways for RNA set1. (B) The network of RSV-correlated pathways for RNA set2. Blue and green represent RNAs that are significantly down-regulated in the samples in the acute phase of infection. Red and orange represent RNAs that are significantly up-regulated in the samples in the acute phase of infection. represent the overlapped pathways. 


\section{Table 1 (on next page)}

Table 1. The number and type of RNAs involved in each module. 
1 Table 1. The number and type of RNAs involved in each module.

\begin{tabular}{llll}
\hline Module color & Number of RNAs & Number of IncRNAs & Number of mRNAs \\
\hline black & 124 & 11 & 113 \\
blue & 217 & 5 & 212 \\
brown & 215 & 1 & 214 \\
green & 206 & 3 & 203 \\
greenyellow & 88 & 6 & 82 \\
grey & 379 & 17 & 362 \\
magenta & 110 & 3 & 107 \\
pink & 122 & 1 & 121 \\
purple & 91 & 2 & 89 \\
red & 182 & 15 & 167 \\
salmon & 71 & 6 & 65 \\
tan & 80 & 6 & 74 \\
turquoise & 394 & 8 & 386 \\
yellow & 210 & 19 & 191 \\
\hline
\end{tabular}

2 Note: lncRNA, long non-coding RNA.

3

4

5

6

7

8

9

10 


\section{Table 2 (on next page)}

Table 2. The Gene Ontology (GO) terms (A) and pathways (B) separately enriched for the mRNAs in RNA set1 and RNA set2. 
1 Table 2. The Gene Ontology (GO) terms (A) and pathways (B) separately enriched for the 2 mRNAs in RNA set1 and RNA set2. FDR, false discovery rate.

\begin{tabular}{|c|c|c|c|c|}
\hline Category & Term & Count & P-value & FDR \\
\hline \multirow[t]{17}{*}{ RNA set1 } & GO:0006350 transcription & 155 & $8.10 \mathrm{E}-12$ & $1.81 \mathrm{E}-08$ \\
\hline & GO:0046649 lymphocyte activation & 34 & $2.86 \mathrm{E}-11$ & $3.20 \mathrm{E}-08$ \\
\hline & GO:0045449 regulation of transcription & 178 & 7.42E-11 & $5.54 \mathrm{E}-08$ \\
\hline & GO:0045321 leukocyte activation & 34 & $5.22 \mathrm{E}-09$ & $2.92 \mathrm{E}-06$ \\
\hline & GO:0030098 lymphocyte differentiation & 21 & $1.52 \mathrm{E}-08$ & $6.81 \mathrm{E}-06$ \\
\hline & GO:0042110 T cell activation & 23 & $2.25 \mathrm{E}-08$ & 8.41E-06 \\
\hline & GO:0001775 cell activation & 35 & 1.07E-07 & $3.42 \mathrm{E}-05$ \\
\hline & GO:0030217 T cell differentiation & 15 & $5.89 \mathrm{E}-07$ & $1.65 \mathrm{E}-04$ \\
\hline & GO:0002521 leukocyte differentiation & 21 & $9.30 \mathrm{E}-07$ & 2.31E-04 \\
\hline & GO:0030097 hemopoiesis & 27 & $1.27 \mathrm{E}-05$ & $2.84 \mathrm{E}-03$ \\
\hline & GO:0006355 regulation of transcription, DNA-dependent & 113 & 2.25E-05 & 4.57E-03 \\
\hline & GO:0048534 hemopoietic or lymphoid organ development & 28 & $2.54 \mathrm{E}-05$ & 4.73E-03 \\
\hline & GO:0002520 immune system development & 29 & $2.77 \mathrm{E}-05$ & 4.76E-03 \\
\hline & GO:0006955 immune response & 54 & 3.95E-05 & 5.89E-03 \\
\hline & GO:0051252 regulation of RNA metabolic process & 114 & 3.70E-05 & $5.91 \mathrm{E}-03$ \\
\hline & GO:0042113 B cell activation & 13 & $1.00 \mathrm{E}-04$ & $1.39 \mathrm{E}-02$ \\
\hline & GO:0002684 positive regulation of immune system process & 24 & $2.89 \mathrm{E}-04$ & 3.73E-02 \\
\hline \multirow[t]{18}{*}{ RNA set2 } & GO:0006952 defense response & 68 & $5.40 \mathrm{E}-17$ & $1.32 \mathrm{E}-13$ \\
\hline & GO:0009611 response to wounding & 55 & $1.48 \mathrm{E}-12$ & $1.81 \mathrm{E}-09$ \\
\hline & GO:0009617 response to bacterium & 29 & $1.73 \mathrm{E}-10$ & $1.40 \mathrm{E}-07$ \\
\hline & GO:0006954 inflammatory response & 36 & 3.71E-09 & $2.26 \mathrm{E}-06$ \\
\hline & GO:0006955 immune response & 57 & 3.78E-09 & $1.84 \mathrm{E}-06$ \\
\hline & GO:0001817 regulation of cytokine production & 24 & $9.50 \mathrm{E}-08$ & $3.86 \mathrm{E}-05$ \\
\hline & GO:0042742 defense response to bacterium & 18 & 3.59E-07 & $1.25 \mathrm{E}-04$ \\
\hline & GO:0006935 chemotaxis & 21 & $8.54 \mathrm{E}-07$ & $2.60 \mathrm{E}-04$ \\
\hline & GO:0042330 taxis & 21 & 8.54E-07 & $2.60 \mathrm{E}-04$ \\
\hline & GO:0007599 hemostasis & 16 & $5.45 \mathrm{E}-06$ & $1.47 \mathrm{E}-03$ \\
\hline & GO:0002237 response to molecule of bacterial origin & 14 & 8.97E-06 & 2.18E-03 \\
\hline & GO:0050817 coagulation & 15 & $1.28 \mathrm{E}-05$ & $2.84 \mathrm{E}-03$ \\
\hline & GO:0007596 blood coagulation & 15 & $1.28 \mathrm{E}-05$ & $2.84 \mathrm{E}-03$ \\
\hline & GO:0042060 wound healing & 21 & $1.33 \mathrm{E}-05$ & 2.69E-03 \\
\hline & GO:0006690 icosanoid metabolic process & 10 & 3.17E-05 & $5.93 \mathrm{E}-03$ \\
\hline & GO:0050878 regulation of body fluid levels & 17 & 3.61E-05 & $6.26 \mathrm{E}-03$ \\
\hline & GO:0033559 unsaturated fatty acid metabolic process & 10 & $6.25 \mathrm{E}-05$ & $1.01 \mathrm{E}-02$ \\
\hline & GO:0032496 response to lipopolysaccharide & 12 & 7.47E-05 & $1.13 \mathrm{E}-02$ \\
\hline
\end{tabular}




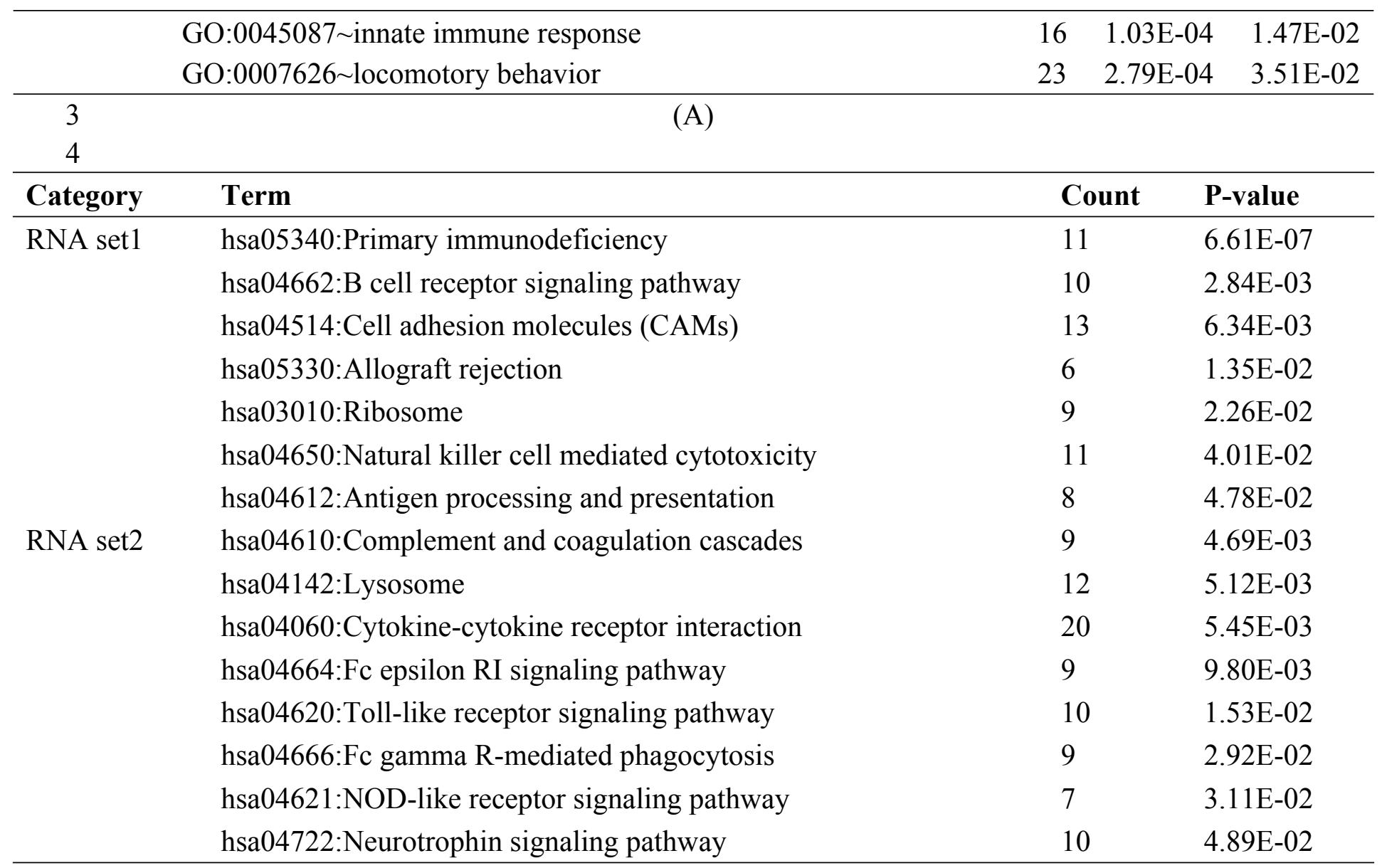

6 FDR, false discovery rate. 


\section{Table 3(on next page)}

Table 3. The pathways enriched for the mRNAs in the co-expression networks for RNA set1 and RNA set2. 
3 Table 3. The pathways enriched for the mRNAs in the co-expression networks for RNA set1 and 4 RNA set2.

\begin{tabular}{llrc}
\hline \multicolumn{1}{c}{ Tategory } & Count & P-value \\
\hline RNA set1 & hsa04640:Hematopoietic cell lineage & 15 & $2.25 \mathrm{E}-06$ \\
& hsa05340:Primary immunodeficiency & 9 & $2.89 \mathrm{E}-05$ \\
& hsa04660:T cell receptor signaling pathway & 15 & $3.41 \mathrm{E}-05$ \\
& hsa04662:B cell receptor signaling pathway & 10 & $1.60 \mathrm{E}-03$ \\
& hsa03010:Ribosome & 9 & $1.41 \mathrm{E}-02$ \\
& hsa04514:Cell adhesion molecules (CAMs) & 9 & $1.12 \mathrm{E}-02$ \\
& hsa04650:Natural killer cell mediated cytotoxicity & 9 & $1.15 \mathrm{E}-02$ \\
& hsa05330:Allograft rejection & 4 & $1.45 \mathrm{E}-02$ \\
& hsa00600:Sphingolipid metabolism & 4 & $1.72 \mathrm{E}-02$ \\
hsa04350:TGF-beta signaling pathway & 6 & $2.15 \mathrm{E}-02$ \\
hsa00480:Glutathione metabolism & 7 & $3.50 \mathrm{E}-03$ \\
hsa04060:Cytokine-cytokine receptor interaction & 17 & $4.80 \mathrm{E}-03$ \\
hsa00600:Sphingolipid metabolism & 6 & $5.70 \mathrm{E}-03$ \\
hsa00052:Galactose metabolism & 5 & $6.90 \mathrm{E}-03$ \\
hsa04142:Lysosome & 10 & $7.90 \mathrm{E}-03$ \\
hsa04621:NOD-like receptor signaling pathway & 7 & $1.00 \mathrm{E}-02$ \\
hsa04640:Hematopoietic cell lineage & 8 & $1.39 \mathrm{E}-02$ \\
hsa00010:Glycolysis / Gluconeogenesis & 6 & $3.26 \mathrm{E}-02$ \\
hsa00500:Starch and sucrose metabolism & 5 & $3.56 \mathrm{E}-02$ \\
hsa00030:Pentose phosphate pathway & 4 & $3.72 \mathrm{E}-02$ \\
hsa00561:Glycerolipid metabolism & 5 & $4.43 \mathrm{E}-01$ \\
\hline
\end{tabular}


Figure 1

Figure 1. The box plots before and after data normalization.

A

Before normalization

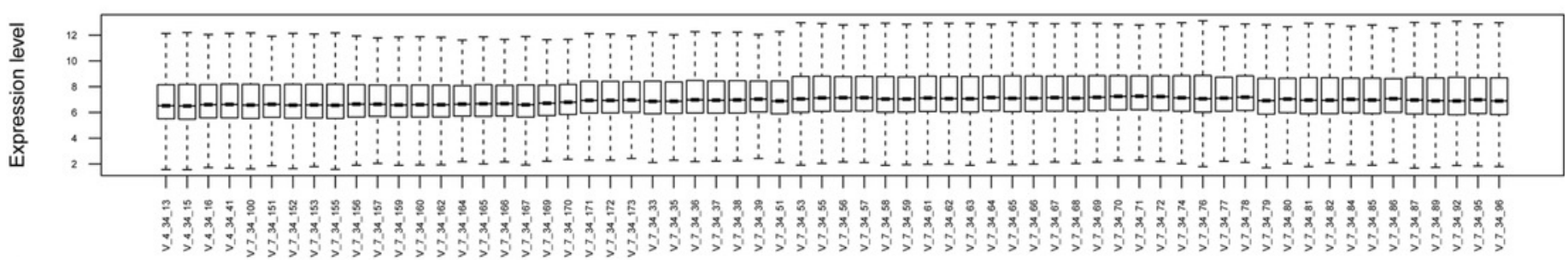

B

After normalization

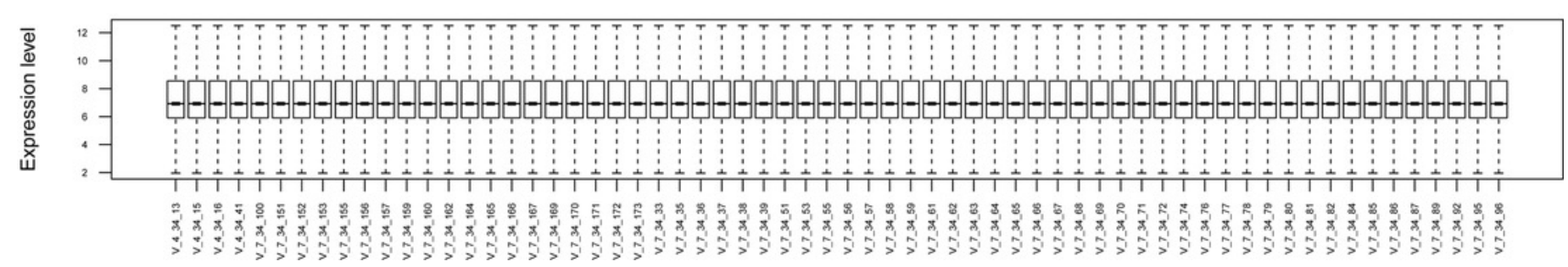


Figure 2 (on next page)

Figure 2. The volcano plot of the differentially expressed RNAs (DE-RNAs).

The green horizontal dashed line represents false discovery rate $(F D R)<0.05$, the two green vertical dashed lines represent $\mid$ log fold change $(F C) \mid>0.263$. The blue and red dots separately represent the significantly down-regulated and up-regulated RNAs in the samples in the acute phase of infection. 


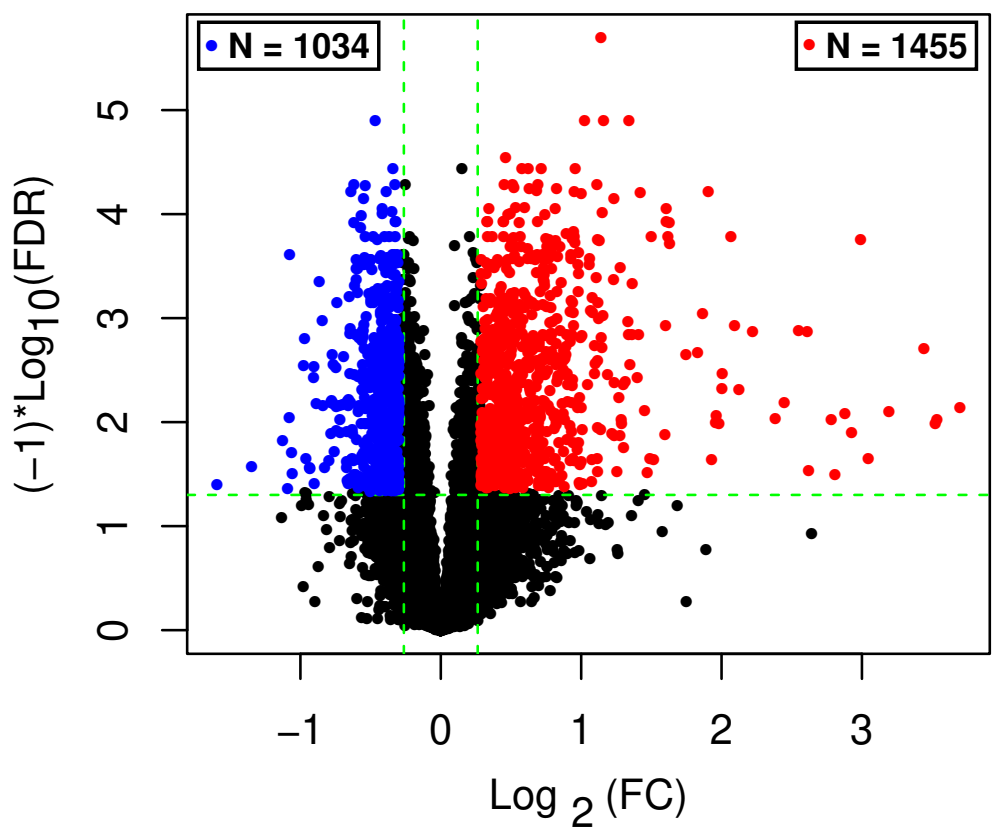


Figure 3

Figure 3. The lustering heatmap based on the expression levels of the differentially expressed RNAs (DE-RNAs).

Red and blue in the sample bar represent the samples in the acute phase of infection and the samples in the recovery period, respectively. 


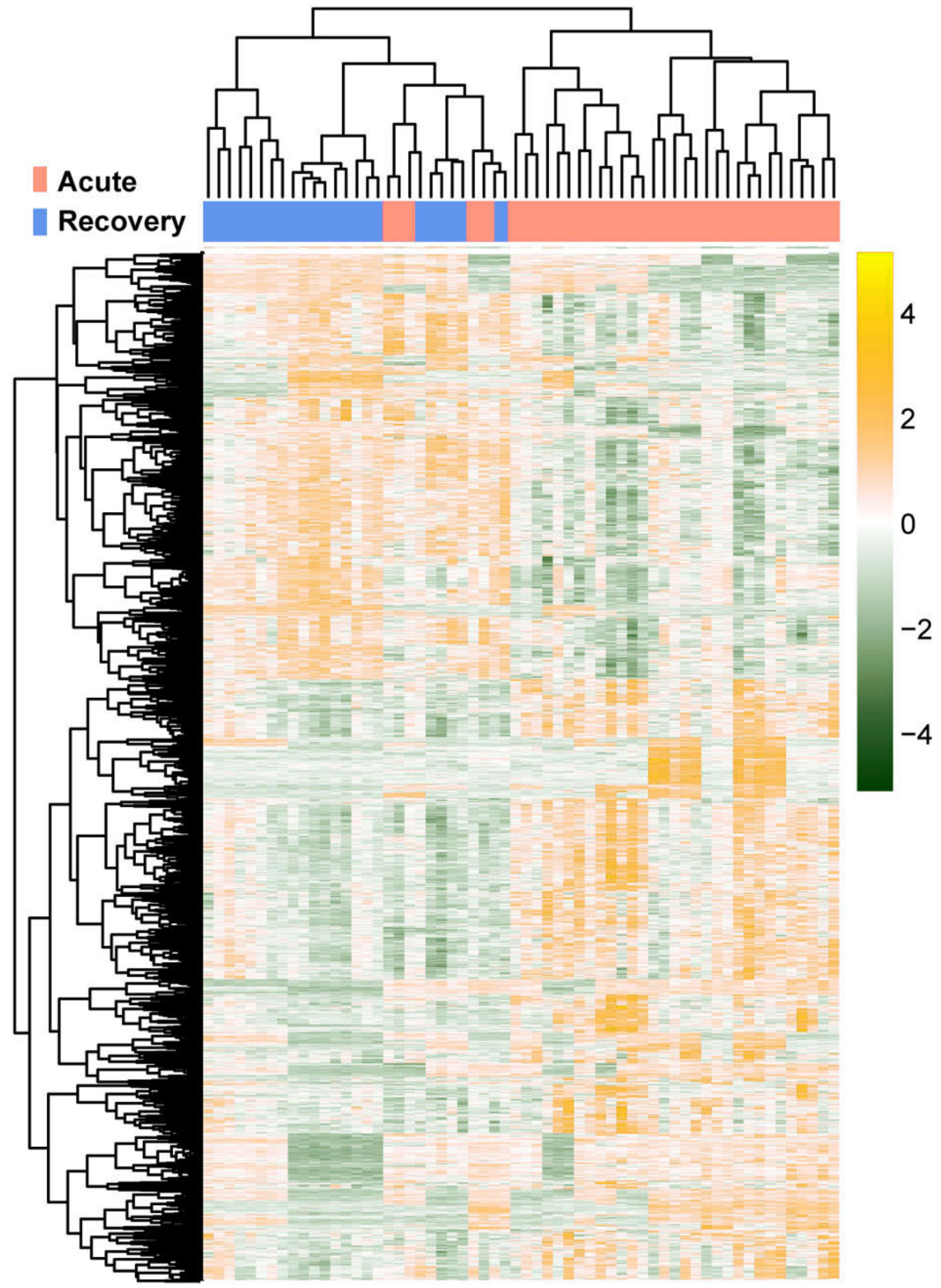

Peer] reviewing PDF | (2019:01:34204:1:1:ACCEPTED 24 Apr 2019) 


\section{Figure 4}

The results of weighed gene co-expression network analysis (WGCNA).

(A) The selection of the weight parameter "power" of adjacency matrix (left diagram; red line is the standard line when the square value of the correlation coefficient achieved 0.9) and the mean connectivity of RNAs (right diagram; red line indicates that the average node degree of the constructed co-expression network is 1 when power = 18); (B) The tree diagram for module division (different color represent different modules); (C) The correlation heatmap for each module and different disease symptoms including disease severity (mild, moderate and severe) and stage (acute and recovery). The numbers in the grids represent correlation coefficients, and the numbers in parentheses represent the significance $p$-values. 
A

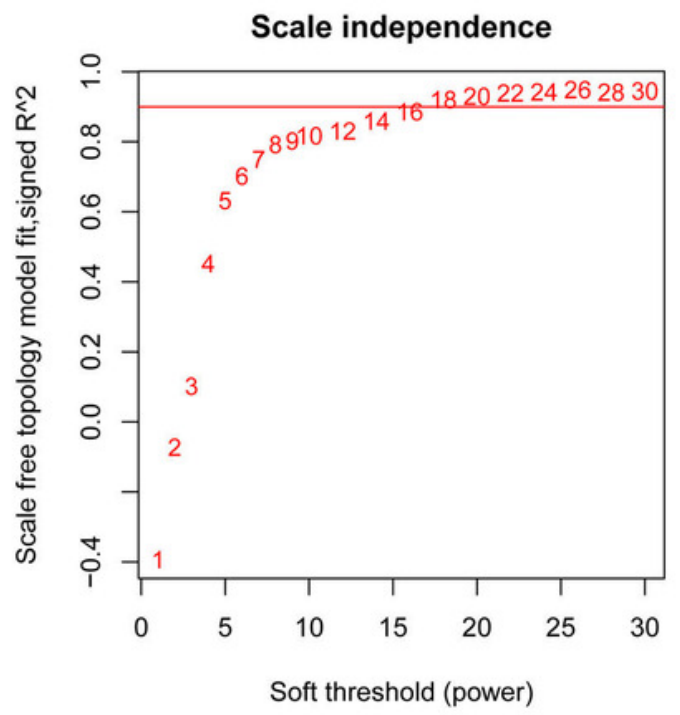

B

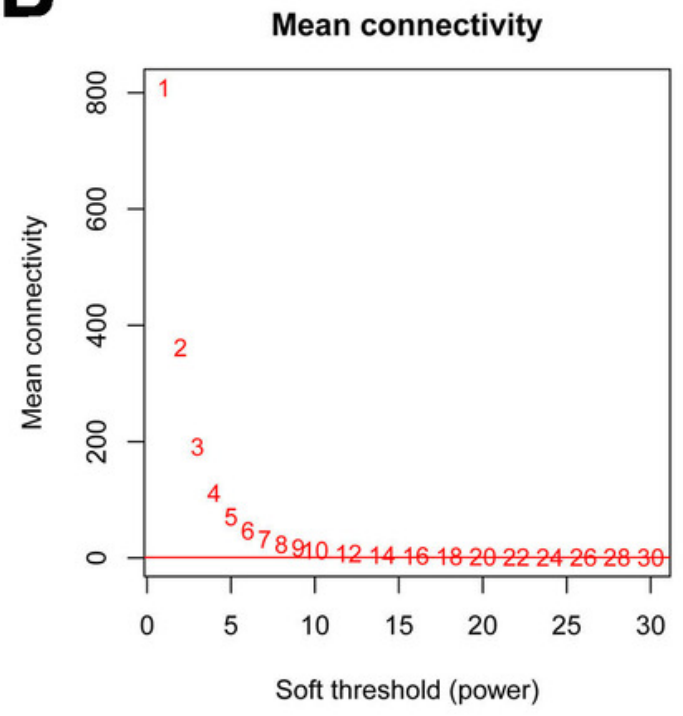

C

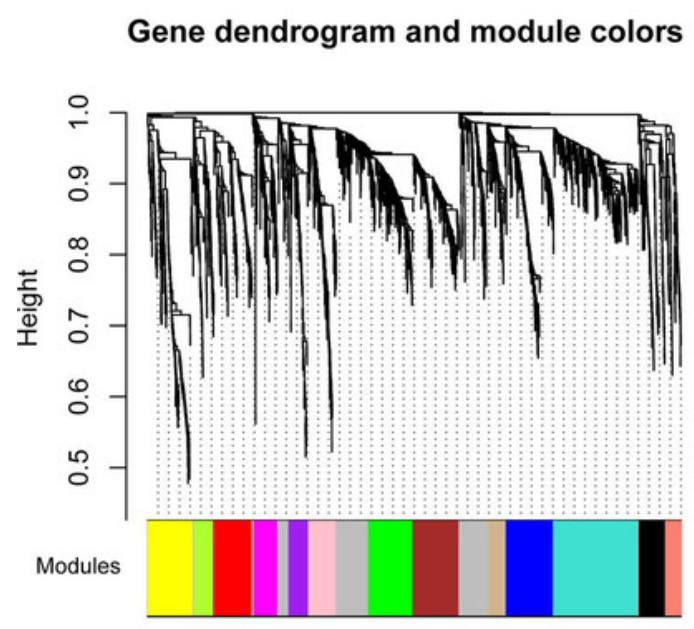

\section{D}

Module-trait relationships

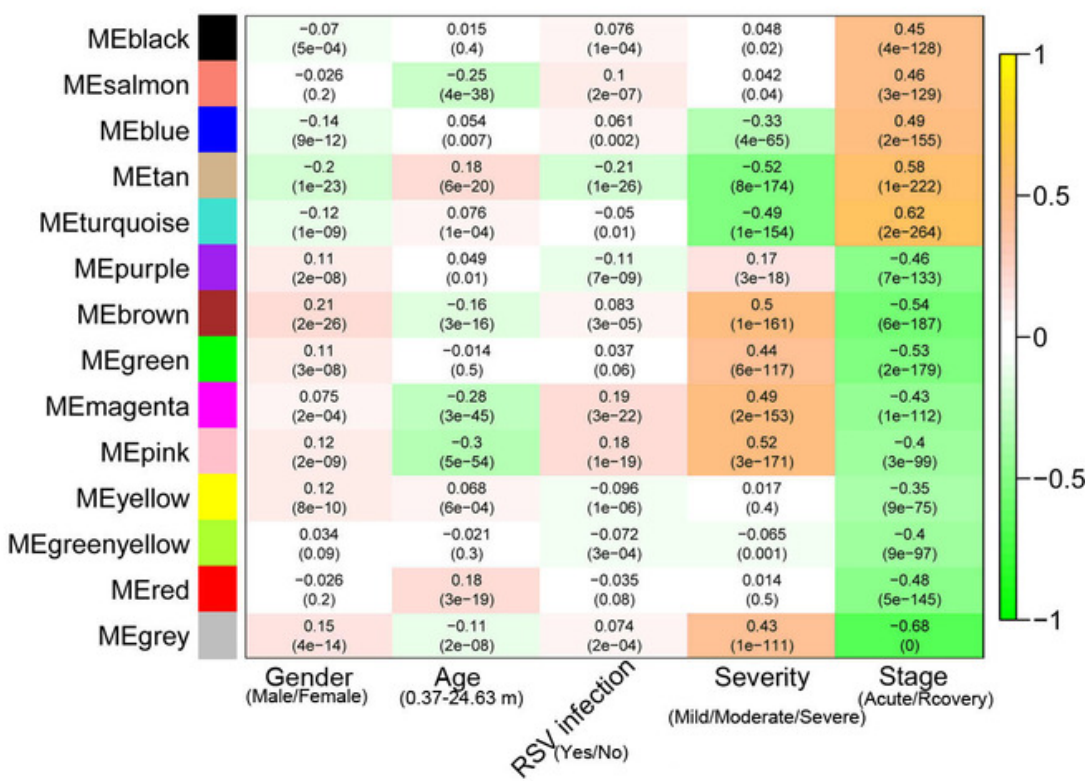


Figure $\mathbf{5}$ (on next page)

Figure 5. Co-expression networks.

(A) The co-expression network for RNA set1. (B) The co-expression network for RNA set2. Blue and green represent RNAs that are significantly down-regulated in the samples in the acute phase of infection. Red and orange represent RNAs that are significantly up-regulated in the samples in the acute phase of infection. Squares and circles separately represent long non-coding RNAs (IncRNAs) and mRNAs. 


\section{PeerJ}

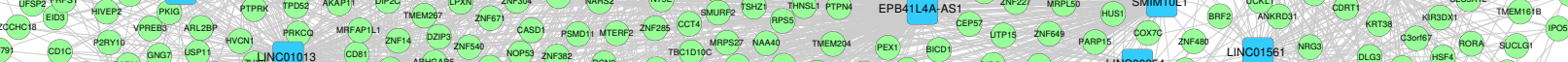

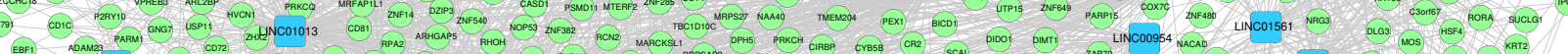

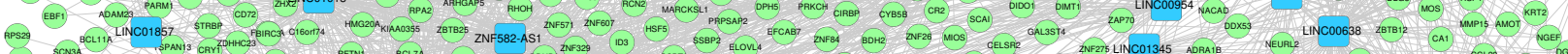

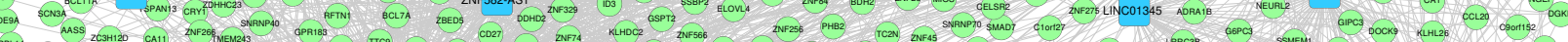

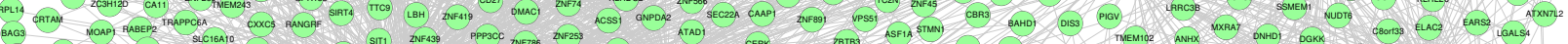

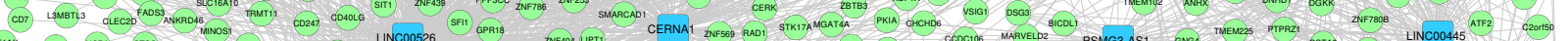

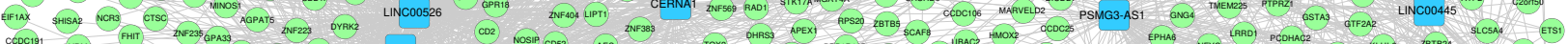

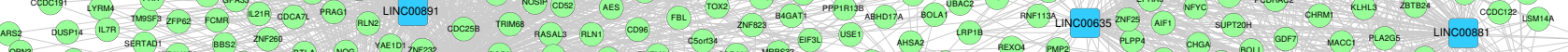

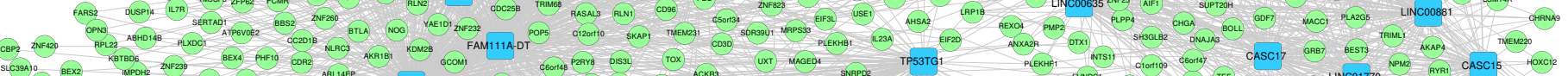

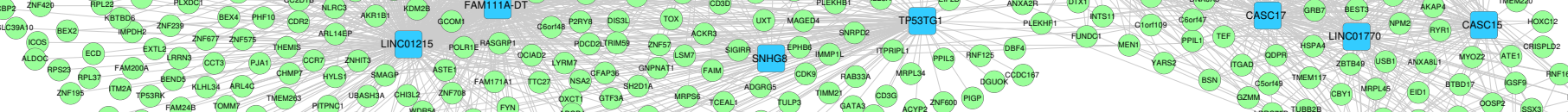

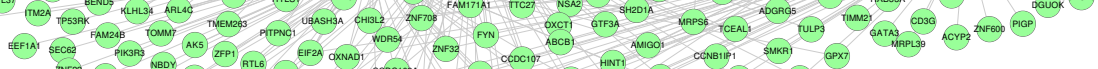

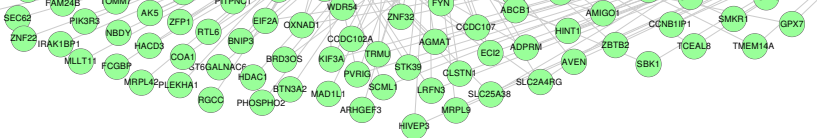

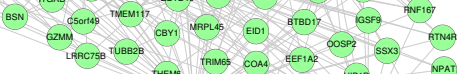

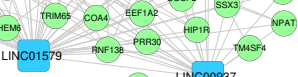

B

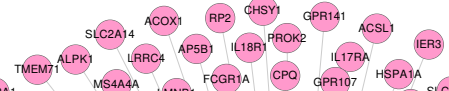

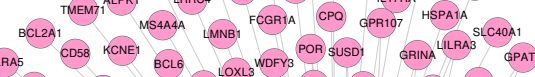

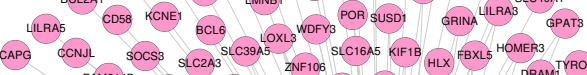

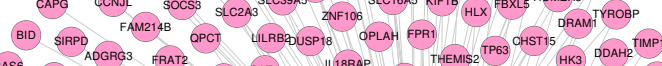

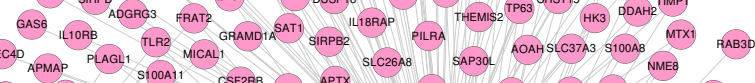

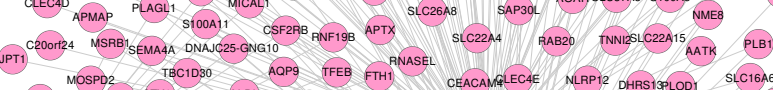

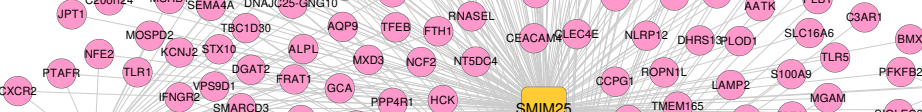

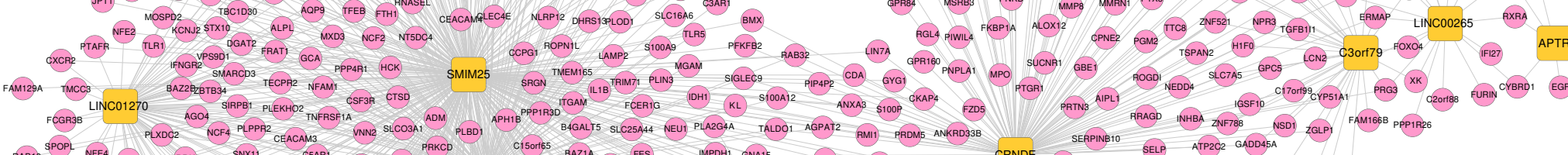

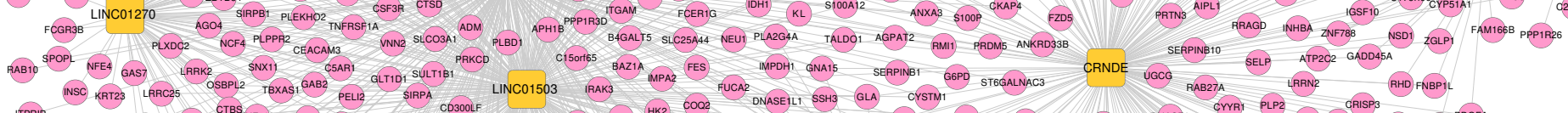

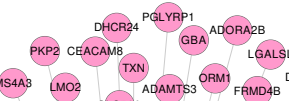

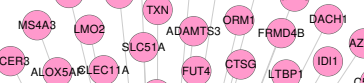

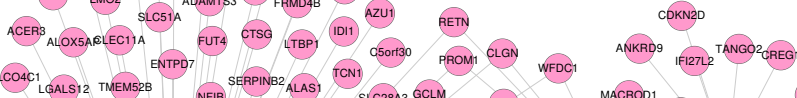

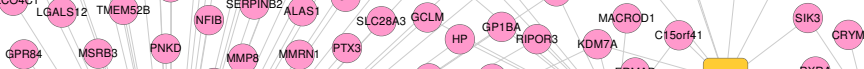

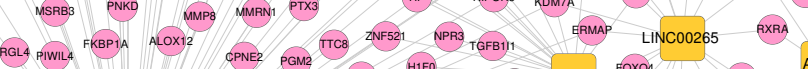
FURIN CYBRD1 EGF EHBPI)

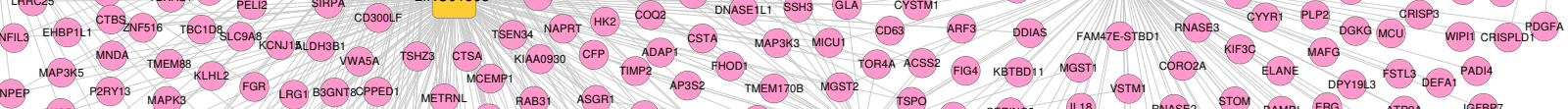
QXXCh)

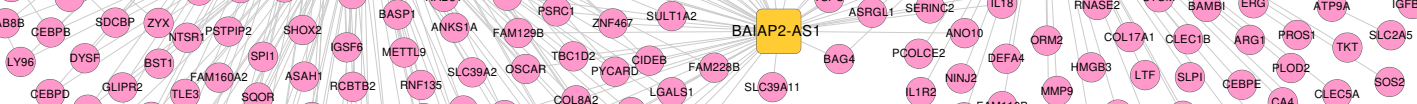

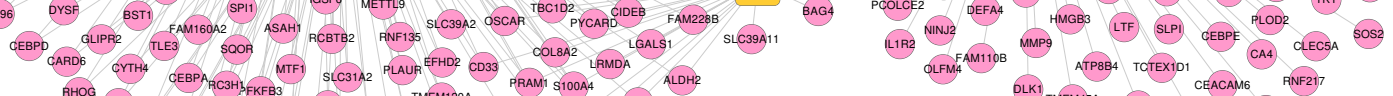

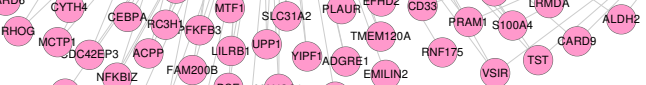

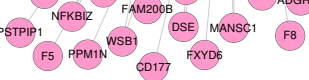

Peer) reviewing PDF | (2019:01:34204:1:1:ACCEPTED 24 Apr 2019) 
Figure 6 (on next page)

Figure 6. Networks of respiratory syncytial virus (RSV)-correlated pathways.

(A) The network of RSV-correlated pathways for RNA set1. (B) The network of RSV-correlated pathways for RNA set2. Blue and green represent RNAs that are significantly down-regulated in the samples in the acute phase of infection. Red and orange represent RNAs that are significantly up-regulated in the samples in the acute phase of infection. Squares and circles separately represent long non-coding RNAs (IncRNAs) and mRNAs. Red diamonds represent the overlapped pathways. 


\section{PeerJ}

FAM111A-DT

Manuscript to be reviewed

HLA-DMA

LINC00926

hsa04514:Cell

CD28

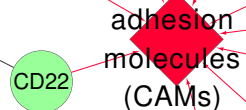

ICOS

HLA-DRA

PPP3CC

NCR3

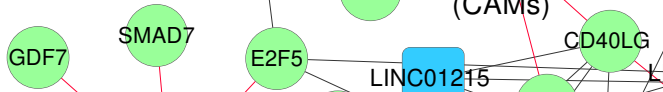

hsa04350:TGF-beta

signaling

pathway

ID3 LINC00526

ITGA6

LINC01013

CD247

CD3D hsa04650:Natural

RASGRP1 hsa04660:T

EBLN3P

LINC00891

SMURF2

EPB41L4A-AS1
ITK

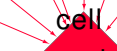

receptor

signaling

pathway

PIK3R3

VAV3

ZAP70

FYN

PSTPIP1 hsa04621iNOD-like

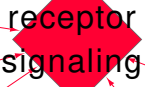

MAPK3

pathway

CARD 9

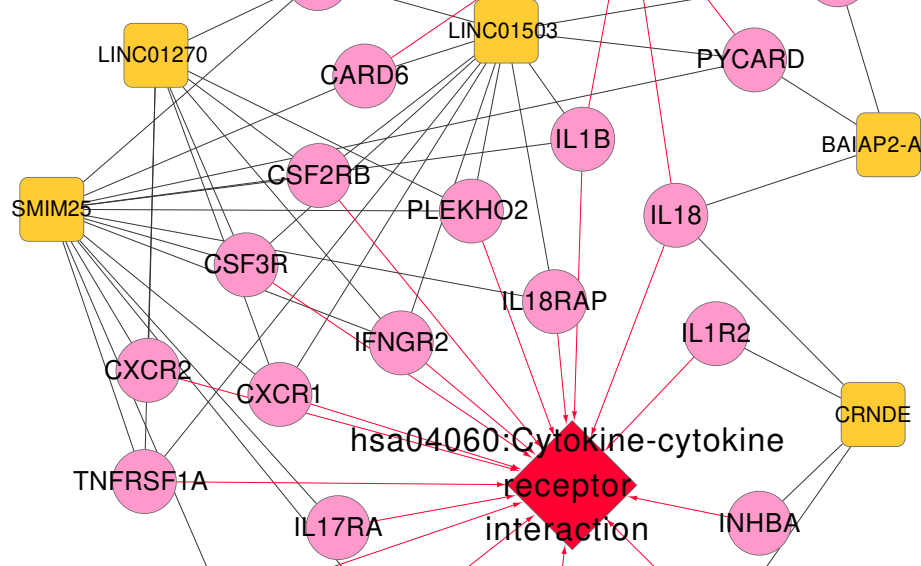

